\title{
Pairwise comparison matrices and the error-free property of the decision maker
}

\author{
József Temesi
}

Published online: 16 April 2010

(C) Springer-Verlag 2010

\begin{abstract}
Pairwise comparison is a popular assessment method either for deriving criteria-weights or for evaluating alternatives according to a given criterion. In realworld applications consistency of the comparisons rarely happens: intransitivity can occur. The aim of the paper is to discuss the relationship between the consistency of the decision maker-described with the error-free property-and the consistency of the pairwise comparison matrix (PCM). The concept of error-free matrix is used to demonstrate that consistency of the PCM is not a sufficient condition of the error-free property of the decision maker. Informed and uninformed decision makers are defined. In the first stage of an assessment method a consistent or near-consistent matrix should be achieved: detecting, measuring and improving consistency are part of any procedure with both types of decision makers. In the second stage additional information are needed to reveal the decision maker's real preferences. Interactive questioning procedures are recommended to reach that goal.
\end{abstract}

Keywords Decision making · Pairwise comparisons · Consistency · Error-free matrices

The research was partly supported by the Hungarian National Research Fund, Grant K 77420.

The first version of this paper was presented at the 19th International Conference on Multiple Criteria Decision Making in Auckland, New Zealand, January 2008. This revised paper used valuable comments from an anonymous reviewer.

J. Temesi $(\varangle)$

Corvinus University of Budapest, Fôvám tér 8, Budapest 1093, Hungary

e-mail: jozsef.temesi@uni-corvinus.hu 


\section{Introduction}

Deriving priorities, weights of criteria, or eliciting preference intensities (scores) of the alternatives according to a given criterion based on the preferences of the decision maker (DM) can be done with various methodological tools. Following the works of Saaty the pairwise comparison approach has been proved as one of the most popular methods in the field of multi-attribute decision making. Having a decision problem with $n$ alternatives and $k$ criteria most of the solution methods use a pay-off matrix and weights for the criteria. In a real world problem it could be difficult to obtain the elements of the pay-off matrix and the values of the weight vector. The AHP method (Saaty 1980) uses pairwise comparison to elicit data, and the hierarchical problem is solved by applying the eigenvector method. Several authors analyzed the AHP, focusing on different elements of the method. It is not the aim of this paper to deal with them, however, AHP and its philosophy serve as references for certain points in our discussion.

If the pairwise comparison matrix (PCM) is given, a great variety of methods have been recommended to estimate the preferences of the DM. Choo and Wedley (2004) and Lin (2007) collected a great number of estimation methods from previous works and they found that the efficiency of the estimation depends on the characteristics of the PCM. If the matrix has "good" properties, e.g. it is a near-consistent matrix, then the estimations from very different type of methods are very close to each other, and the most simple estimation methods can be applied. In that sense the consistency of the PCM is important.

Producing a PCM via the judgments of the DM is a controversial procedure. As most of the researchers agree, if the number of elements to be compared in a pairwise manner is high ( $>6$ or 7 ), the DM is uncertain in her answers and the PCM fails to meet certain requirements, e.g. expressing the real preferences of the DM. We can say that the DM is not able to give us her values consequently. In that sense the consistency of the decision maker is important.

The aim of this paper is to distinguish the consistency of the PCM and the consistency of the decision maker. Using the same word-consistency-in both cases can be controversial. This is why I will reserve the term "consistency" for the PCM, and the decision maker will be labeled with another term: this is the error-free property.

It is essential that I will treat the problem from a decision-aiding approach (Tsoukias 2008). The decision maker and the decision problem are in the front of the discussion.

\section{Pairwise comparisons and decision making}

Suppose, that we want to evaluate $n$ alternatives according to a given criterion. The method is to compare pairs of alternatives, and the values $v_{1}, v_{2}, \ldots, v_{n}$ (preference intensities, scores) are estimated from the $n \times n$ PCM. The problem is the same if we want to determine criteria weights: in that case we have to obtain pairwise comparison values for pairs of criteria. It is common in both cases that the preference intensities or weights are derived from data measured on ratio scale (Fichtner 1986; Wedley 2008). 
We can say that alternative $A_{i}$ is preferred to alternative $A_{j}$ according to the ratio $v_{i} / v_{j}$.

Definition 1 Decision makers can be classified as "informed" and "uninformed". The informed DM knows $v_{1}, v_{2}, \ldots, v_{n}$ explicitly. The uninformed DM may not know $v_{1}$, $v_{2}, \ldots, v_{n}$ sufficiently, or $v_{1}, v_{2}, \ldots, v_{n}$ may not even exist and need to be developed by aiding the DM appropriately.

The DM (in a procedure we do not define at the moment) will give pairwise comparisons $t_{i j}$ to be considered as the approximation of the ratios $v_{i} / v_{j}$ (for all $i, j=1$, $2, \ldots, n, i \neq j)$. The PCM T $=\left[t_{i j}\right]$ is positive reciprocal $\left(t_{i j}>0\right.$ for all $i, j t_{j i}=1 / t_{i j}$ if $i \neq \mathrm{j}$ ), and the diagonal elements are equal to 1 . We have to note that the reciprocal property assumes a consequent behavior of the DM. That assumption makes possible the reduction of the number of pairwise comparisons to a half: we have to ask only for one comparison having two pairs of alternatives with the same elements in different order.

In the rest of the paper further definitions and propositions will follow. Some of the propositions do not need a formal proof, they are consequences of the definitions (see e.g. Proposition 1). Other propositions can describe conjectures (see e.g. Proposition 6).

Definition 2 Matrix $\mathbf{T}$ is error-free if $t_{i j}=v_{i} / v_{j}$ (for all $i, j=1,2, \ldots, n, i \neq j$ ), as defined by Choo and Wedley (2004).

In that case the elements of matrix $\mathbf{T}$ reflect the real preferences of the DM.

Definition 3 Matrix $\mathbf{T}$ is consistent if $t_{i j} \cdot t_{j k}=t_{i k}$, for all $i, j, k=1,2, \ldots, n$. The rank of a consistent matrix $\mathbf{T}$ is 1 .

This is the well-known transitivity property for the triads of alternatives.

Proposition 1 If $\mathbf{T}$ is error-free, then $\mathbf{T}$ is consistent.

Proposition 1 is a straight consequence of Definitions 2 and 3.

The preference intensities will be derived from $\mathbf{T}$ with the help of the $\Phi$ estimation function (Choo and Wedley 2004). The properties of the estimation methods have been thoroughly analyzed (see e.g.Cogger and Yu 1985; Golany and Kress 1993). Let the result of the estimation be $\mathbf{w}$.

Proposition 2 If $\mathbf{T}$ is error-free, then $\mathbf{w}=\mathbf{v}$.

The proposition is a consequence of the interpretation of $\mathbf{v}$.

In case of an error-free $\mathbf{T}$, estimating the values of $\mathbf{w}$ can be done with several equivalent methods. In this case the properties of the chosen method will not influence the result, so it is worth to choose the most simple method (e.g. estimation from the columns of $\mathbf{T}$ ).

\section{Error-free property of the decision maker and the consistency of the pairwise comparison matrix}

Definition 4 If the elements of $\mathbf{T}$ are given on ratio scale, the decision maker is errorfree if 
1. the PCM is a positive, reciprocal, consistent matrix,

2. the pairwise comparisons reflect precisely to the decision maker's real preferences.

Proposition $\mathbf{3}$ The decision maker is error-free if and only if $\mathbf{T}$ is error-free.

The usual inconsistency measures (see later) are based on the consistency of the PCM. They do not say anything about the error-free property of the decision maker. It should be emphasized that the DM is the only person, who may know and who can give information about the real values of $v_{1}, v_{2}, \ldots, v_{n}$. An inconsistent $\mathbf{T}$ matrix can signal the error caused by an informed DM, but it can often happen, too, that the DM (informed or uninformed) is unable to give an error-free matrix via pairwise comparisons. We can define different inconsistency measures for $\mathbf{T}$, and correction methods can be built to reach a consistent or a near-consistent matrix. The importance of distinguishing the two types of the DMs is that the decision aiding procedure has to reflect to it.

Saaty and others put the emphasis on the fact that to find decision makers, who will give a consistent $\mathbf{T}$, is practically impossible. In real world decision situations the matrix $\mathbf{T}$ of a decision maker will contain intransitivity.

\section{Proposition 4 DM likely produces inconsistent $\mathbf{T}$.}

What are the sources of inconsistency? Decision scientists stress that-especially with a great number of alternatives - the DM could not see the consequences of so many pairwise comparisons: psychological, cognitive limitations are the obstacles. Even in case of an informed DM, revealing preferences can cause errors in the pairwise questioning procedure. It is rarely emphasized that the source of inconsistency can be the questioning procedure itself, or in special cases the applied scaling leads to a built-in inconsistency (e.g. any scaling system with fixed endpoints). Assuming an uninformed DM, revealing uncertain preferences would cause the inconsistency, too.

Most of the practitioners do not analyze the reasons leading to inconsistent matrix $\mathbf{T}$. The consequence is that a consistent or a near-consistent PCM is accepted for the estimation of $\mathbf{v}$ even in case of an uninformed DM, without further analysis. This approach can result in values $v_{i}$ far from the real ones.

Proposition 5 It is possible to have consistent $\mathbf{T}$ that is not error-free.

Remark Choo and Wedley (2004) discuss this proposition in an other context.

In case of an uninformed DM, consistency of PCM is not sufficient for reaching the error-free property: the (2) condition of the error-free DM should be met, too.

It is possible, for instance, that we have more than one consistent matrix to the same problem: only one of them can be error-free.

Example 1 Suppose that we obtained the following matrix from the decision maker:

$$
\mathbf{T}=\left[\begin{array}{lll}
1 & 2 & 5 \\
& 1 & 3 \\
& & 1
\end{array}\right]
$$


Looking at the matrix we can see that it is not transitive (not consistent). Suppose that we want to give the possibility to the DM to correct the matrix with modifying one element only. Our recommendation for the DM is to choose one of the matrices as follows:

$$
\mathbf{T}_{1}=\left[\begin{array}{lll}
1 & 2 & 6 \\
& 1 & 3 \\
& & 1
\end{array}\right] \quad \mathbf{T}_{2}=\left[\begin{array}{ccc}
1 & 2 & 5 \\
& 1 & 5 / 2 \\
& & 1
\end{array}\right] \quad \mathbf{T}_{3}=\left[\begin{array}{ccc}
1 & 5 / 3 & 5 \\
& 1 & 3 \\
& & 1
\end{array}\right]
$$

If the error-free matrix is one of the $\mathbf{T}_{1}, \mathbf{T}_{2}, \mathbf{T}_{3}$ matrices, then without having additional information from the DM we are not able to tell which one is the "correct" matrix $\mathbf{T}$ (as $\mathbf{v}$ is not known).

We can say that in this particular case we do not need a correction. In the first step we can accept the original $\mathbf{T}$ as a good approximation of a consistent matrix, and we can get the $\mathbf{w}$ values from a proper $\Phi$ estimation function. In the second step the DM will check the w vector, and if she accepts it, then the procedure is over. This argumentation assumes an inconsistency measure (indicator, index), and a threshold value to decide whether $\mathbf{T}$ is accepted or not.

However, even if we can measure the inconsistency, and we have a threshold value, we can construct an example, where the original $\mathbf{T}$ cannot be accepted, even though our choice problem of $\mathbf{T}$ is relevant.

In Example 1 we could not solve a correction problem, because the connection between the consistency of the $\mathbf{T}$ matrix and the error-free property of the DM, as it is declared in Proposition 5, does not allow us to follow the logic of choosing any consistent matrix.

Consistency of the $\mathbf{T}$ matrix is a necessary (but not sufficient) condition to get an error-free matrix T. However, if we want to have a close to error-free matrix we have to know "how far" we are from a consistent matrix (simply because the error-free matrix is consistent). Therefore, we have to face with the problem of the measurability of inconsistency of PCMs.

Definition 5 Matrix $\mathbf{T}$ is near-consistent if the value of a given inconsistency measure (indicator) is lower than a threshold value.

Before taking a glance at detecting, measuring and improving consistency of the $\mathbf{T}$ matrix, I want to clarify the significance of the hypothesis that in most cases $\mathbf{v}$ is not known explicitly.

\section{Proposition 6 Decision makers in real-life decision situations are uninformed.}

Two examples are provided. There are famous cases in the literature that have been constructed in the light of a known vector $\mathbf{v}$. I will demonstrate that their interpretations can be misleading. The illustrative cases are mutations of certain Saaty examples: to draw the consequences the exact copies of the examples are not necessary.

Example 2 Suppose that the task is to compare the volumes of different fruits. Estimating the volumes is not easy because of the shape of the fruits, but it is possible to measure them with a slight error. The measurement says that the ratios of the volume 
of three fruits are 1:4:8. A perfectly informed decision maker can give a consistent matrix with those ratios:

$$
\mathbf{T}_{\mathrm{F}}=\left[\begin{array}{lll}
1 & 4 & 8 \\
& 1 & 2 \\
& & 1
\end{array}\right]
$$

Three persons were requested to make the comparisons, and we got the following matrices:

$$
\mathbf{T}_{\mathrm{F} 1}=\left[\begin{array}{lll}
1 & 4 & 7 \\
& 1 & 3 \\
& & 1
\end{array}\right] \quad \mathbf{T}_{\mathrm{F} 2}=\left[\begin{array}{lll}
1 & 2 & 9 \\
& 1 & 3 \\
& & 1
\end{array}\right] \quad \mathbf{T}_{\mathrm{F} 3}=\left[\begin{array}{lll}
1 & 2 & 6 \\
& 1 & 3 \\
& & 1
\end{array}\right]
$$

Without knowing the measured volumes and supported only with the inconsistency measures of the matrices we may conclude that $\mathbf{T}_{\mathrm{F} 3}$ is acceptable, and the third person is an error-free DM, because she has a consistent matrix. Unfortunately, the real volumes can show us, that none of the DMs was error-free, and from that very rationale viewpoint none of them gave the real ratios. We can even convince them, of course, about their "wrong" judgments allowing them to see the measured values.

Our question is: given the three matrices from three uninformed DMs (no opportunity to measure the volumes), do we need any correction for any of the matrices? If yes, what would be the basis of the correction?

Example 3 For the sake of simplicity, let suppose that after asking three persons about the wealth of three nations with the method of pairwise comparisons, we have got the same matrices, as in Example 2. Let the ratios of GDP be 1:4:8, having the same $\mathbf{T}_{\mathrm{F}}$ as before. What can we say about the consistency of the "decision makers"?

The wealth of nations is not objectively measurable, it is influenced by several factors, GDP is a usual proxy measure. The first and the second person would realize, that they did not give transitive answers compared to the known GDP values, however, they could insist that the $\mathbf{w}_{\mathrm{F} 1}$ and $\mathbf{w}_{\mathrm{F} 2}$ values obtained from their matrices $\mathbf{T}_{\mathrm{F} 1}$ and $\mathbf{T}_{\mathrm{F} 2}$, and different from the $\mathbf{v}_{\mathrm{F}}$ values estimated from the matrix $\mathbf{T}_{\mathrm{F}}$, can describe their preferences properly!

It is possible that we will accept (after some adjustment) all three matrices: all of the decision makers with different $\mathbf{T}$ matrices are error-free! We have three different $\mathbf{v}$ vectors, each of them differs from the $\mathbf{v}_{\mathrm{F}}$ vector, but they are in total harmony with their preferences, and reflect precisely the subjective evaluations of the decision makers about the wealth of nations.

In real group decision making situations with subjective preferences it can often happen, that we ask for pairwise comparisons of the same alternatives according to a given criterion, and in that case we might face the result what we have got in Example 3.

Saaty uses these examples (Saaty 1980, 2005) to demonstrate the validity of the pairwise comparison method for rationale decision makers: they are able to give "good" estimations. The resolution of the controversy is that Saaty solves a different problem: approximation of a known $\mathbf{v}$ vector with pairwise comparisons. Knowing the correct values we can evaluate the DM (her "consistency"), too. In real cases, where $\mathbf{v}$ is 
unknown, we do not have reference vectors, and the DM reveals her hidden preferences via pairwise comparisons. Who can judge the result? Only the decision maker herself. In a decision aiding process the consultant can see the consistency or inconsistency of the PCM, and he can call the attention of the decision maker to that kind of inconsistency; but he is not able to show the right direction of any improvement, simply because he has no idea about it.

Proposition 7 In case of unknown $\mathbf{v}$, improving the consistency of an inconsistent matrix is possible only with additional information from the decision maker.

\section{Detecting, measuring and improving consistency}

In real-world decision processes, where PCM approaches are used, the following steps can be applied:

Step 1: Determining the elements of the pairwise-comparison matrix

Step 2: Consistency analysis

Step 3: Correction of the PCM

Step 4: Applying an estimation method

Step 5: Verification

This 5-step procedure was analyzed in Temesi (2006) focusing on the consistency problem. What are the general reasons leading us to detect and to measure inconsistency in Step 2?

- It could be crucial to check the aptitude of the DM (e.g. in group decision making), when excluding him/her from the decision process is possible: PCMs very far from being consistent can signal the ineligibility of the DM.

- The analysis of the given problem could be resulted in claiming that pairwise comparison as a modeling tool could not be applied.

- The most common reason is that inconsistency measure is supposed to measure the degree of intransitivity, and with the modification of matrix $\mathbf{T}$ we want to help the decision maker to be more consequent.

Most of the authors respect Saaty's consistency index and consistency ratio as references when they create their own indicators. Saaty uses the difference $\left(\lambda_{\max }-n\right)$ to define a consistency ratio. His consistency ratio compares $\left(\lambda_{\max }-n\right) /(n-1)-$ the consistency index, CI-, with an index, RI, calculated from the same values from randomly generated reciprocal matrices. Saaty says that if CI/RI $>0.1$, the DM should revise her judgments on preferences.

The advantages of the Saaty ratio within the logic of his method are obvious. However, critiques say that the $10 \%$-rule is an arbitrary rule of thumb, near-consistent pairwise comparison matrices not having accepted by the $10 \%$-rule can be constructed, and some intuitively "far from consistent" matrices might be accepted. We can add that in the applications the consistency of the $\mathbf{T}$ matrix is regarded here as the consistency of the DM.

While constructing new consistency measures (indices) the researchers more or less accepted a few properties for those measures: 
- in case of a consistent matrix the value of the index should be 0 ,

- upper and lower bounds are needed (the lower bound in general is 0),

- the values of the index between the lower and upper bound should have an interpretation from a consistency viewpoint, or at least a threshold value could be verified,

- if the scale is limited in a questioning procedure, the upper bound of the consistency index could be given using the lower and upper values of the scale.

There are several approaches to measuring consistency. The harmonic consistency index (Stein and Mizzi 2007) is based on the additive normalization method. Aguarón and Moreno-Jiménez (2003) created the geometric consistency index using the estimator of the variance of the perturbations of Crawford and Williams (1985) from the row geometric mean method. Koczkodaj (1993) used the concept of triads for measuring consistency. Bozóki and Rapcsák (2008) developed the idea further and they proved a relationship between the Saaty ratio and the Koczkodaj index. This short list of the approaches suggests that a generally accepted method for measuring the inconsistency of a PCM does not exist. Saaty's inconsistency index dominates the applications, however, its interpretation is fragile, especially regarding the threshold value.

In order to get an error-free matrix not only the properties of the original PCM is crucial, but the accuracy and the efficiency of the estimation function, too. The estimation function $\Phi$ is efficient, if $\Phi(\mathbf{T})$ is "close enough" to the real preference vector. The concept of efficiency is different from the accuracy of the estimation. Choo and Wedley (2004) and Lin (2007) have investigated 18 estimation methods for the pairwise comparison problem. 12 of the methods were distance minimizing method, using the distance of $w_{i} / w_{j}$ from $t_{i j}$ (e.g. least square, least absolute error, logarithmic least square). In case of error-free matrices they analyzed six methods (e.g. simple normalized column sum, right eigenvector, preference weighted geometric mean). The conclusion is that neither the accuracy of the estimation (fitness), nor the consistency ratio of a PCM will measure the error-free property of the decision maker, because those indicators can express only one dimension of that property.

In the light of our discussion the straightforward application of the 5-step procedure and the role of certain steps should be analyzed further. Determining the elements of the PCM and verification of the results are the most critical steps. Consistency is a necessary condition, and it should be checked, however, the significance of the consistency analysis and its application together with a consistency improvement method are different, depending on the type of the decision maker.

\section{Correcting the elements of PCM for the informed and uninformed DM}

Suppose that in Step 2 we chose a method to measure the consistency of matrix $\mathbf{T}$. If matrix $\mathbf{T}$ is consistent or near-consistent we would go to Step 4, and we have to check the error-free property in Step 5.

If matrix $\mathbf{T}$ is not consistent, then consistency improvement is needed in Step 3. The improvement method will correct some (or all) elements in matrix $\mathbf{T}$ in order to obtain a new matrix with better consistency. Applying a correction method it is crucial to know what kind of error has been occurred during the elicitation method (Step 1): 
Type 1: The errors, fluctuations in the judgments of the DM were around the "correct" values within a small distance; there is a kind of "noise" in the system.

Type 2: Systematic errors, under- or over-estimations of certain comparisons occurred.

Type 3: Outliers could be detected, e.g. as a consequence of communication errors or misunderstandings.

According to the type of the error various improvement techniques can be developed:

- improvement can be done with a perturbation method (in case of noise),

- improving the consistency of a sequence of triads (in case of under- or over-estimation),

- adjustment starting from the greatest violence of consistency (in case of outliers).

In case of errors with Type 1 the assumption is that all elements can be biased, and an increase in consistency can be achieved by the modification of (almost) all elements of matrix T. The method of Xu and Wei (1999) developed for the AHP is a good example of that approach.

If certain comparisons contain judgment errors-Type 2-then systematic analysis of the triads of matrix $\mathbf{T}$ and replacing some elements with their corrected values can lead to better or perfect consistency. Bozóki et al. (2010) use integer programming to detect the number of triads and to locate inconsistency. Their method does not even need an inconsistency measure: after determining the minimum number of elements to be modified to meet the perfect consistency a procedure is given to execute the modifications.

The most common approach is to locate the greatest violence of consistency in a matrix T. Modifying the proper element in a good direction the consistency of matrix $\mathbf{T}$ will be better. The AHP applications follow that approach (Saaty 2005), but approaches with totally different philosophy, for instance the singular value decomposition method (Gass and Rapcsák 2004) can determine corrections on the same way.

Having an inconsistent $\mathbf{T}$ and an informed DM, the correction method can help eliciting an error-free matrix, because the informed DM has a quite good knowledge of preference intensities of $v_{j}$. Having an inconsistent $\mathbf{T}$ and an uninformed DM the error-free property is not guaranteed by the consistency or near-consistency of $\mathbf{T}$ having been reached by the correction method; it must be incorporated into the decision aiding process.

The correction methods differ in requiring the presence of the decision maker. There are approaches that do not need the DM in the improvement procedure. The absence of the DM can be explained by various reasons:

- The DM is busy and she has no time or she is not available for an other questioning.

- The task is an ex post analysis of a decision has been made earlier, the decision makers are not available.

- The PCM was built from empirical facts without meeting the DM personally.

Proposition 8 Applying automatic correction methods (i.e. no information is given by the DM) in Step 3 is possible, but in that case the presence of the DM is required in Step 5. 
The best way to obtain error-free PCM is to ensure the permanent presence of the DM in the entire decision making procedure. Eliciting the elements of $\mathbf{T}$ and improving the consistency in an interactive process can lead to one of the following results:

- an error-free matrix $\mathbf{T}$ is accepted,

- it is impossible to get an error-free matrix for the given problem.

Proposition 9 Interactive methods for generating matrix $\mathbf{T}$ are invariant to the type of the DM.

The consequence of Proposition 9 is that using interactive procedures Step 1 (and Step 3, if it is applied) can replace Step 5. Proposition 9 suggests that in real-world applications we can avoid the verification problem applying interactive methods. However, in other cases explicit use of a verification method is needed to get an error-free matrix.

Proposition 10 If the decision maker is not present in the correction method, the resulted $\mathbf{T}$ (and $\mathbf{w}$ ) should be verified in Step 5.

A possible verification is to prove that the given problem belongs to the class of Type 1, 2 or 3, and the applied automatic correction method is relevant to that type.

In certain cases (when the elicitation of the elements of the PCM leads to a consistent T) the whole process is reduced to two stages: generating PCM and the estimation of $\mathbf{w}$. The proposed approach is to obtain a consistent or near-consistent PCM with a proper interactive questioning procedure before estimating the $\mathbf{w}$ vector.

The questioning procedure would meet the following conditions:

a) the DM must participate in the entire procedure (availability),

b) the DM reveals preferences without any influence (neutrality),

c) the DM is supported to discover and to reveal her real preferences (decisionaiding).

Based on $(\mathrm{a}-\mathrm{c})$ several procedures can be built to reach the matrix $\mathbf{T}$ at the end of the first stage. A few options are listed here differing in their strategy:

A) Multiple random questioning: the DM is requested to repeat the elicitation procedure from different initial pairs of alternatives on different pathways (Temesi 2006).

B) Reducing the number of questions with ensuring transitivity in each step.

C) Combining different elicitation techniques (Srdjevic 2005).

D) Combining the elicitation with mathematical programming (goal-programming) models (Shirland et al. 2003).

Having a consistent or a near-consistent matrix, the choice of $\Phi$ is not crucial after the first stage, the properties of the estimation methods become important only in case of a PCM far from a consistent matrix. One of the most significant problems is rank reversal, which is discussed in the literature exhaustively. A more strict condition, the preservation of order of intensity of preference is described by Bana e Costa and Vansnick (2008): if there is a ranking relation between $t_{i j}$ and $t_{k l}$, then the $w_{i} / w_{j}$, $w_{k} / w_{l}$ ratios should also satisfy that relation. Most of the common estimation methods do not meet that very restrictive criterion. 


\section{Conclusion}

In multiattribute decision making problems using pairwise comparison matrices it is crucial to distinguish the type of the decision maker. Obtaining an error-free PCM will require different approaches if we have informed or uninformed decision maker. Most of consistency and verification problems can be avoided with the application of interactive procedures ensuring the presence of the decision maker in each step. These procedures can contain various combinations of simple questioning, consistency checking and estimation methods.

\section{References}

Aguarón J, Moreno-Jiménez JM (2003) The geometric consistency index: approximated thresholds. Eur J Oper Res 147:137-145

Bana e Costa CA, Vansnick J-C (2008) A critical analysis of the eigenvalue method used to derive priorities in AHP. Eur J Oper Res 187:1422-1428

Bozóki S, Rapcsák T (2008) On Saaty's and Koczkodaj’s inconsistencies of pairwise comparison matrices. J Glob Optim 42(2):157-175

Bozóki S, Fülöp J, Poesz A (2010) On pairwise comparison matrices that can be made consistent by the modification of a few elements. Cent Eur J Oper Res. doi:10.1007/s10100-010-0136-9

Choo EU, Wedley WC (2004) A common framework for deriving preference values from pairwise comparison matrices. Comput Oper Res 31:893-908

Cogger KO, Yu PL (1985) "Eigenweight vectors and least distance approximation for revealed preference in pairwise weight ratios". J Optim Theor Appl 46:483-491

Crawford G, Williams C (1985) A note on the analysis of subjective judgment matrices. J Math Psychol 29:387-405

Fichtner J (1986) On deriving priority vectors from matrices of pairwise comparisons. Socioecon Plann Sci 20:341-345

Gass SI, Rapcsák T (2004) “Singular value decomposition in AHP”. Eur J Oper Res 154:573-584

Golany B, Kress M (1993) "A multicriteria evaluation of methods for obtaining weights from ratio-scale matrices”. Eur J Oper Res 69:210-220

Koczkodaj WW (1993) A new definition of consistency of pairwise comparisons. Math Comput Model 18(7):79-84

Lin C-C (2007) A revised framework for deriving preference values from pairwise comparison matrices. Eur J Oper Res 176:1145-1150

Peláez JI, Lamata MT (2003) A new measure of consistency for positive reciprocal matrices. Comput Math Appl 46:1839-1845

Saaty TL (1980) The analytic hierarch process. McGraw Hill, New York

Saaty TL (2005) The analytic hierarchy and analytic network processes for the measurement of intangible criteria and for decision-making. In: Figueira J, Greco S, Ehrgott M (eds) Multiple criteria decision analysis: state of the art surveys. Springer, New York pp 345-407

Shirland LE, Jesse RR, Thompson RL, Iacavou CL (2003) Determining attribute weights using mathematical programming. Omega 31:423-437

Srdjevic B (2005) Combining different prioritization methods in the analytic hierarchy process synthesis. Comput Oper Res 32:1897-1919

Stein WE, Mizzi PJ (2007) The harmonic consistency index for the analytic hierarchy process. Eur J Oper Res 177:488-497

Temesi J (2006) Consistency of the decision maker in pairwise comparisons. Int J Manage Decis Mak 7:267-274

Tsoukias A (2008) From decision theory to decision aiding methodology. Eur J Oper Res 187:138-161

Wedley WC (2008) From no thing to some things_-deriving ratio preferences. 19th International Conference on MCDM, Auckland, pp 32-33

Xu Z, Wei C (1999) A consistency improving method in the analytic hierarchy process. Eur J Oper Res 116:443-449 